Nervenarzt 2020 $91: 303-311$ https://doi.org/10.1007/s00115-020-00882-4 Online publiziert: 16. März 2020

(c) Der/die Autor(en) 2020

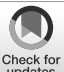

\author{
A. Mühlbäck ${ }^{1,2} \cdot$ K. S. Lindenberg ${ }^{1} \cdot$ C. Saft ${ }^{3} \cdot$ J. Priller $^{4} \cdot$ G. B. Landwehrmeyer ${ }^{1}$ \\ ${ }^{1}$ Abteilung Neurologie, Universitätsklinikum Ulm, Ulm, Deutschland \\ ${ }^{2}$ Klinik für Neurologie und Zentrum für klinische Neurowissenschaften, 1. Medizinische Fakultät, \\ Karlsuniversität, Prag, Tschechien \\ ${ }^{3}$ Huntington-Zentrum NRW, Neurologische Klinik der Ruhr-Universität Bochum, St. Josef-Hospital, \\ Bochum, Deutschland \\ ${ }^{4}$ Klinik für Psychiatrie und Psychotherapie, Charité Universitätsmedizin Berlin, Berlin, Deutschland
}

\title{
Genselektive Therapieansätze bei der Huntington-Krankheit
}

Wiederholungssequenzen innerhalb des ersten Exons des HTT-Gens aufChromosom 4 verursacht. Bei mehr als 39 CAGWiederholungen besteht eine vollständige Penetranz. Typischerweise kommt es im mittleren Lebensalter zur klinischen Manifestation der HK, wobei eine inverse Korrelation zwischen der Anzahl an CAG-Triplett-Wiederholungen und dem Erkrankungsbeginn besteht $[1,2]$.

In Europa leiden mehr als 12 Personen pro 100.000 Einwohner an der HK und etwa die dreifache Anzahl von Individuen tragen ein $50 \%$ iges Risiko, die Genmutation vom betroffenen Elternteil geerbt zu haben [3]. Die HK ist somit eine seltene Erbkrankheit, die allerdings für diese Krankheitskategorie vergleichsweise viele Menschen betrifft. Da immer mehr Deutsche ein hohes Lebensalter erreichen und somit lange genug leben, um eine klinische Manifestation der HK auch bei geringen CAG-Triplett-Wiederholungen (36-39) im HTT-Gen zu erlauben, ist eine Zunahme der Prävalenz wahrscheinlich [4].

Anlageträger für die HTT-Gen-Expansionsmutation (HDGEM, „huntington's diseases gene expansion mutation") sind in den ersten Lebensjahren typischerweise klinisch und funktionell nicht von Personen unterscheidbar, die keine HDGEM-Träger sind. Erst im Verlauf entwickeln Anlageträger motorische Symptome (z. B. unwillkürliche, choreatiforme Bewegungen), kognitive Beeinträchtigungen und Verhaltensauffälligkeiten, die eine zunehmende Beeinträchtigung im Alltag zur Folge haben.
Die HK ist eine progrediente neurodegenerative Erkrankung, die im Verlauf zur Pflegebedürftigkeit und zum frühzeitigen Tod führt, typischerweise etwa zwei Jahrzehnte nach dem Beginn diagnoseweisender motorischer Symptome [5]. Der Verlauf der HK lässt sich deskriptiv in prämanifeste und manifeste Stadien unterteilen, wobei nach dem Schweregrad der funktionellen Beeinträchtigung ein beginnendes, mittelgradiges und fortgeschrittenes Stadium differenziert werden kann.

\section{\) Die HK führt im Verlauf zur Pflegebedürftigkeit und zum frühzeitigen Tod}

Für Personen, die zwar eine positive Familienanamnese, jedoch keine klinischen Symptome oder Anzeichen für eine HK aufweisen, ist ein prädiktiver Gentest möglich. HDGEM-Träger weisen typischerweise bereits 10 bis 15 Jahre vor dem Auftreten klinischneurologischer Symptome in der Magnetresonanztomographie (MRT) einen Volumenverlust im Corpus striatum auf. Zudem können sie subtile, aber reproduzierbar nachweisbare Veränderungen im Verhalten, in der Kognition und der Motorik entwickeln (Prodromalstadium der HK; $[6,7])$.

Die Verfügbarkeit verlässlicher, prädiktiver Gentests erlaubt konzeptuell die Prävention einer manifesten Erkrankung bei rechtzeitigem Einsatz krankheitsmodifizierender Therapien. Die jüngsten sche Expansion von CAG-Trinukleotid- 


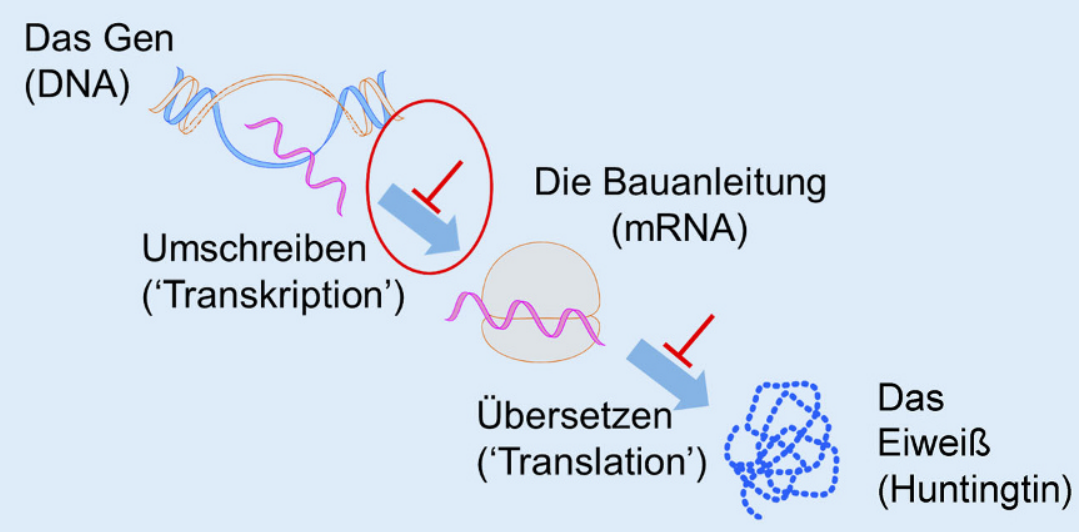

Abb. 1 ॥ Die verschiedenen Ansatzpunkte der genselektiven Behandlungsstrategien bei der Huntington-Krankheit

Fortschritte bei Gen-silencing/loweringTechniken, wie beispielsweise das ASO RG6042 (Ionis/Hoffmann-LaRoche) zur verminderten Genexpression, ermöglichten die ersten klinischen Studien mit HK-Patienten im Frühstadium der Erkrankung und erbrachten den Nachweis, dass die lumbale intrathekale Applikation des Wirkstoffs zu einer dosisabhängigen Reduktion von HTT im Liquor cerebrospinalis führt [8].

\section{Pathogenese der Huntington- Krankheit: ein Überblick}

Die genetische Ursache der HK ist seit 1993 bekannt, jedoch sind die molekularen pathogenetischen Mechanismen, die zur selektiven und für die HK typischen Neurodegeneration insbesondere im Striatum führen, immer noch nicht vollständig verstanden. Zahlreiche molekulare und zelluläre Mechanismen der HK-Pathogenese sind diskutiert worden, darunter die Toxizität des mutierten Poly-Glutamin-expandierten Huntingtin (mHTT) bzw. aminoterminaler Fragmente von mHTT mit Aggregation von Poly-Glutamin-expandierten mHTTFragmenten im Zytoplasma und Zellkern [9-11] sowie ein partieller Verlust physiologischer Funktionen des HTTProteins in Folge der Mutation [12].Das mHTT und dessen Spaltprodukte führen aufgrund bisher noch nicht im Detail verstandener molekularer Mechanismen zur Schädigung und zum Verlust von striatalen Projektionsneuronen („medium spiny neurons“, MSNs), von kortikalen Projektionsneuronen und anderen Zellen des Zentralnervensystems (ZNS).

Durch Studien an Modellorganismen und an HK-Patienten wurden zahlreiche Pathomechanismen identifiziert, die bei der komplexen Pathogenese der HK eine Rolle spielen. Hierzu zählen Transkriptionsveränderungen, Alterationen der glutamatergen Transmission mit N-Methyl-D-Aspartat(NMDA)-Rezeptor-Dysregulation, Störungen der $\mathrm{Ca}^{2+}$ abhängigen Signaltransduktion, Störung der $\mathrm{K}^{+}$-Homöostase und sowie Defizite im zellulären Energiestoffwechsel durch Veränderungen der mitochondrialen Funktion. Diese Krankheitsmechanismen sind funktionell miteinander verbunden. Die diagnoseweisenden klinischen Beschwerden bei Patienten mit manifester HK (z.B. Chorea und Dystonie) reflektieren eine Dysfunktion der Basalganglien bzw. kortikostriatothalamischer Schaltkreise.

\section{„Gene silencing“" zur Verminderung der Neusynthese mutanter HTT-Gen-Produkte}

Vielversprechende Behandlungsansätze sind auf eine Hemmung der Genexpression gerichtet, bei der die Produktion des mHTT-Proteins verringert wird. In wegweisenden Studien von Yamamoto et al. und Wang et al. [13, 14] führte die Hemmung der Nachbildung von mHTT im Striatum transgener Mäuse zu Ver- besserungen der Krankheitssymptome. Eine Hemmung der Nachbildung von mHTT-Gen-Produkten kann erreicht werden durch

- den Abbau von mRNA-Transkripten oder

- die Hemmung der Transkription [15].

In $\bullet$ Abb. 1 sind schematisch die Ansatzpunkte genselektiver Therapieansätze bei der HK dargestellt. Diese Ansätze können entweder einen transienten (Monate) oder langfristigen (Jahre) Effekt erzielen. Bislang wurden eine Vielzahl von HTTSilencing-Substanzen aufSicherheit, Verträglichkeit und Wirksamkeit in präklinischen Studien untersucht. Zudem befinden sich gegenwärtig mehrere Substanzen in der Testung in klinischen Studien.

\section{Behandlung mit intrathekal applizierten Medikamenten: ASOs}

Die Behandlung von HK-Patienten mit Antisense-Oligonukleotiden (ASOs) wird aktuell in mehreren klinischen Studien in Deutschland erprobt. ASOTherapeutika stellen eine neue Klasse von Substanzen dar, die eine wirksame und effiziente Modulation der Genexpression in vivo ermöglichen [16]. ASOs binden entsprechend des Watson-CrickBasenpaarungsprinzips an die komplementären Sequenzen der Prä-mRNA und mRNA und führen - präferenziell über RNaseH1 im nukleären Kompartiment zu einem Abbau von HTT-Transkripten $[17,18]$. Da ASOs die Blut-Hirn-Schranke nicht passieren können, müssen sie intrathekal verabreicht werden.

Therapeutisch eingesetzte ASOs können in erster Linie an die mutante HTTmRNA binden („allelselektiv“) oder „nichtallelselektiv“ an Transkripte beider Allele. Das nichtallelselektive HTTASO Ionis- $\mathrm{HTT}_{\mathrm{RX}}$ (auch bekannt als RG6042) erwies sich in einer PhaseIb/IIa-Studie generell als sicher und reduzierte die Spiegel von mHTT im Liquor cerebrospinalis dosisabhängig durchschnittlich um etwa $40 \%$ [8]. Wave Biosciences führt zwei randomisierte, doppelblinde, placebokontrollierte klinische Studien der Phase Ib/IIa 
Hier steht eine Anzeige.

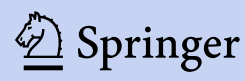


(Precision-HD-1 und -2) durch, um die Sicherheit und Verträglichkeit zweier allelselektiver ASOs, WVE-120101 und WVE-120102, zu bewerten, die an "single nucleotide polymorphisms“ (SNPs) andocken, welche nur auf mutanten HTT-mRNAs zu finden sind (rs362307 bzw. rs362331; [19]). Da sich die SNPs in verschiedenen ethnischen Gruppen unterscheiden, erlauben die ausgewählten SNPs eine kumulative allelselektive Behandlungsabdeckung für ca. $60 \%$ Patienten mit der HK/HDGEM [20].

Die verschiedenen ASOs, die sich in der Entwicklung zur Anwendung bei der HK befinden, verringern die Genexpression durch Degradationsmechanismen (- Abb. 2). ASOs haben durch chemische Modifikationen [21] eine verlängerte Halbwertszeit und eine lipophilere Oberfläche mit verbesserter Gewebepenetration. Sie senken die Genexpression für 3 bis 4 Monate, wirken aber nur vorübergehend, da sie selbst mit der Zeit von DNAsen abgebaut werden. Eine regelmäßig wiederholte Verabreichung ist somit notwendig, um eine anhaltende Wirkung zu erzielen [22].

\section{Divalente siRNA als eine innovative Methode}

Eine Repression der Genexpression im gesamten Gehirn durch kleine interferierende RNAs (siRNA) ist bisher noch nicht erreicht worden. Präklinische Studien zeigen, dass durch die Injektion divalenter siRNA (di-siRNA), aus zwei vollständig chemisch modifizierten und verbundenen siRNAs in das Ventrikelsystem, eine ausgeprägte, 50 - bis $90 \%$ ige Reduktion der HTT-Expression im ZNS erreicht werden kann, auch ohne Verwendung viraler Vektoren [23]. Bei Verabreichung an Makaken zeigte der Ansatz eine gute und gleichmäßige Verteilung über die beiden Hemisphären und führte zu einer deutlichen nichtallelselektiven Reduktion des HTT. Erste präklinische Studien sprechen dafür, dass Allelselektivität auch bei relativ geringer CAG-Länge (36-45) mit dieser Methode erreicht werden kann. Klinische Studien (Phase I/IIb) sind in Vorbereitung (•Tab. 1).

Nervenarzt 2020 $91: 303-311$ https://doi.org/10.1007/s00115-020-00882-4

(c) Der/die Autor(en) 2020

A. Mühlbäck · K. S. Lindenberg · C. Saft · J. Priller · G. B. Landwehrmeyer Genselektive Therapieansätze bei der Huntington-Krankheit

\section{Zusammenfassung}

In Deutschland leiden derzeit mindestens 8000 , vermutlich aber sogar bis zu ca. 14.000 Menschen an einer klinisch apparenten („manifesten") Huntington-Krankheit (HK). Zudem tragen schätzungsweise 24.000 Deutsche die der HK zugrunde liegende Mutation im Huntingtin-(HTT)-Gen und werden im Laufe ihres Lebens an der HK erkranken. Obwohl die HK eine seltene neurodegenerative Erkrankung ist, steht sie gegenwärtig im Fokus eines allgemeinen medizinischen Interesses: Klinische Studien, die eine rationale Basis für die Hoffnung bilden, das bislang unaufhaltsame, schicksalhafte Fortschreiten der Erkrankung bis zur vollständigen Pflegebedürftigkeit bremsen und - bei rechtzeitigem Behandlungsbeginn - eventuell sogar die klinische Manifestation der HK mitigieren zu können, haben begonnen. Diese innovativen Therapieansätze sind darauf ausgerichtet, die Nachbildung mutierter HTT-Gen-Produkte zu hemmen. Eine erste klinische Arzneimittelprüfung zum Nachweis der Wirksamkeit (Phase III) intrathekaler Antisense-Oligonukleotide (ASO, Wirkstoff RG6042) hat 2019 begonnen. Klinische Studien zu weiteren, alternativen Behandlungsansätze mit allelselektiven ASOs sowie zu gentherapeutischen Ansätzen mit RNA-Molekülen und Zinkfinger-RepressorKomplexen stehen kurz bevor. In dem vorliegenden Artikel geben wir einen Überblick über die gegenwärtig diskutierten genselektiven Therapieansätze bei der HK.

\section{Schlüsselwörter}

Huntingtin · Antisense-Oligonukleotid · Gentherapie - Zinkfinger-Repressor-Komplexe . Adeno-assoziierte Viren

\section{Gene-selective treatment approaches for Huntington's disease}

\section{Abstract}

In Germany at least 8000 and probably up to ca. 14,000 people currently suffer from clinically manifest Huntington's disease (HD). In addition, an estimated 24,000 Germans carry the HD mutation in the huntingtin (HTT) gene and will develop HD during their lifetime. Although $\mathrm{HD}$ is a rare neurodegenerative disease, it is currently in the focus of general medical interest: clinical trials have begun that provide a rational basis for hope to slow down the so far relentless progression of the disease, ultimately resulting in patients becoming entirely dependent on nursing care. If treatment is started early enough it may be possible to mitigate the clinical manifestation of HD. These innovative therapeutic approaches aim at inhibiting the de novo production of mutant HTT gene products. A first clinical drug trial to demonstrate the efficacy (phase III) of intrathecal antisense oligonucleotides (ASO, active substance RG6042) was started in 2019. Additional clinical studies on alternative treatment approaches with alleleselective ASOs as well as gene therapeutic approaches using RNA molecules and zinc finger repressor complexes are imminent. This article gives an overview of the current geneselective therapeutic approaches in HD under discussion.

Keywords

Huntingtin - Antisense oligonucleotide - Gene therapy - Zinc finger repressor complexes . Adeno-associated viruses

\section{Behandlung mit oral} verfügbaren Medikamenten: translationsgekoppelter mRNAAbbau

Oral verfügbare, niedermolekulare Substanzen können die Neubildung von mHTT durch vermehrten Abbau der mRNA hemmen, wahrscheinlich durch translationsgekoppelten RNA-Abbau
$[15,24]$. Diese Substanzen erlauben eine Behandlung des gesamten Körpers, nicht nur des ZNS. Nach aktuellem Wissensstand beschränkt sich die HKPathologie nicht nur auf das ZNS, sondern betrifft auch den restlichen Körper, insbesondere die Skelettmuskulatur [25]. Die Entwicklung von HTT-ExpressionsModulatoren wird z.B. von den Pharmaunternehmen PTC Therapeutics [26] 


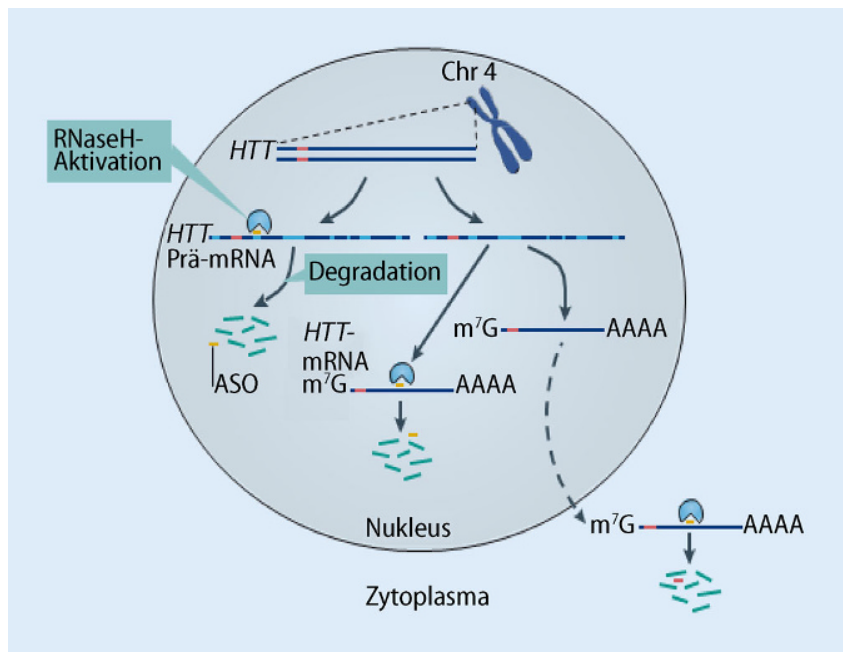

Abb. $2 \triangleleft$ Antisense-Oligonukleotide: Schema. ASO Antisense-Oligonukleotid, Chr4 Chromosom 4, HTT Huntingtin. (Nach [15])

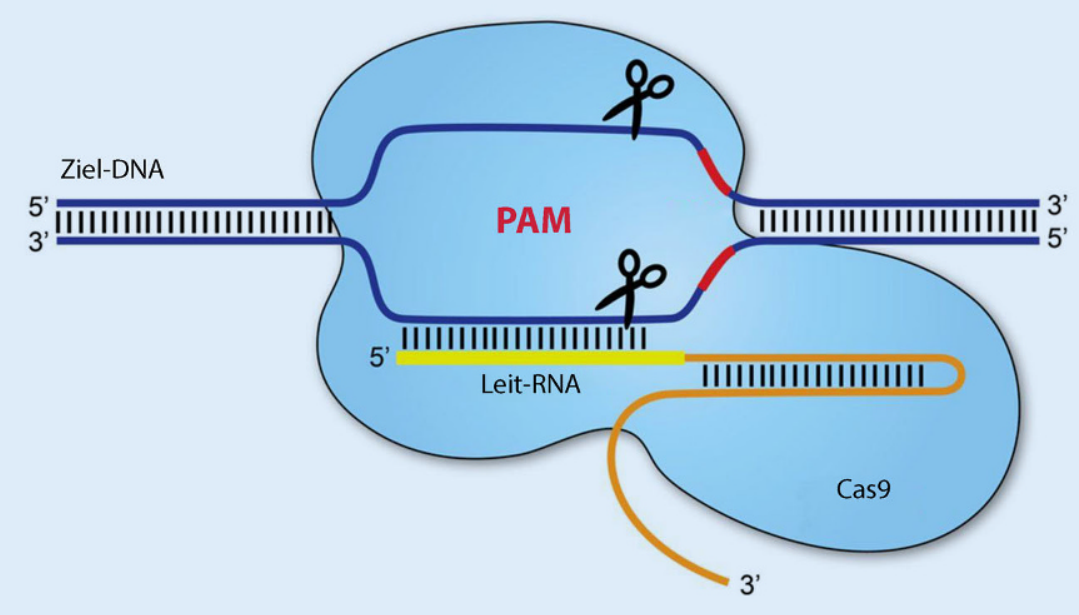

Abb. $3 \Delta$ Das CRISPR/Cas-System mit der sog. „single guide RNA“ (Leit-RNA), die mit einer komplementären DNA-Sequenz (Ziel-DNA) gekoppelt werden kann. Cas9 "CRISPR associated protein" (CRISPR-assoziertes Protein), PAM "protospacer adjacent motif" (eine DNA-Sequenz in der Nähe eines Protospacers). (Nach [38], CC-BY 4.0 http://creativecommons.org/licenses/by/4.0/)

und Novartis vorangetrieben, analog zu der Behandlung der spinalen Muskelatrophie(SMA) mit Risdiplam (RG7916), einem oral verfügbaren Spleißmodulator, der gegenwärtig in klinischen Zulassungsstudien zur Behandlung der SMA erprobt wird.

\section{Gentherapie mit mRNA als Ansatzpunkt}

RNA-Interferenz (RNAi) ist ein natürlicher Prozess in eukaryoten Zellen, der zur spezifischen Herunterregulation der Genexpression („gene silencing") führt. Das therapeutische Ziel der RNAi-Targeting-Ansätze ist es, die Produktion mu- tanter Genprodukte zu hemmen. Hierbei werden vor allem Konstrukte aus Adeno-assoziierten Viren (AAV; [27]), aber auch Lentiviren [28] als Vektoren verwendet, um eine langanhaltende Produktion von $\mathrm{mi}($, ,micro“ $) /$ siRNA im transduzierten Gehirnparenchym zu induzieren. Die Herstellung von Virusvektoren zur Anwendung beim Menschen ist erprobt und gilt als sicher. Darüber hinaus sind AAV in der Lage, postmitotische Zellen wie Neurone zu transfizieren [29].

Die intraparenchymatöse Injektion erfolgt stereotaktisch in Vollnarkose. Im Jahr 2019 gab die Firma UniQure bekannt, von der U.S. Food and Drug Administration (FDA) eine Zulassung zur klinischen Erprobung von ATM-130 (AAV5-miHTT) erlangt zu haben, was in Modellsystemen der HK sowohl die mutierte HTT-mRNA als auch das HTTProtein in allen erfolgreich transduzierten Hirnregionen deutlich reduziert [30]. UniQure zeigte eine dosisabhängige Verteilung von AAV5-miHTT im Hirnparenchym; die Sicherheit und Verträglichkeit dieses nichtallelselektiven Therapieansatzes soll ab 2020 bei Patienten in frühen Stadien der HK geklärt werden.

Der Gentherapieansatz der Firma Voyager besteht aus einem Adeno-assoziierten viralen Kapsid (AAV1) und einem patentgeschützten Transgen (VYHTT01), welches den RNA-InterferenzWeg verwendet, um HTT-mRNA zu eliminieren oder zu reduzieren. Eine direkte intraparenchymatöse Verabreichung von VY-HTT01 ins Gehirn (derzeit bevorzugt: intrastriatale und intrathalamische stereotaktische Injektion) ist im Kontext einer Phase-Ib-Studie geplant.

Langanhaltende Effekte zeigen ebenfalls Behandlungsstrategien mit synthetischer miRNA [31] und "short hairpin RNA“ (shRNA) [32]. Diese modulieren durch eine Interaktion mit dem „RNAinduced silencing complex" (RISC) den mehrstufigen Prozess des endogenen Abbaus reifer mRNA im Zytoplasma.

\section{HTT-Inaktivierung mit- tels CRISPR/Cas9 als Geneditierungswerkzeug}

Mit der Einführung der molekularen Genschere CRIPSR/Cas erlebt die Molekularbiologie zurzeit die größte Revolution seit Jahrzehnten. Das CRISPR/CasSystem wurde von Charpentier und Doubna in Bakterien als Abwehrmechanismus gegen Viren beschrieben [33] und kann zur Editierung des Genoms von Säugetieren eingesetzt werden [34]. Das System verwendet eine RNA-Sequenz, die sog. „single guide RNA“, die an eine komplementäre genomische DNA-Sequenz bindet und mithilfe des Enzyms Cas9 DNA an definierten Stellen schneidet, was zu einer Inaktivierung ganzer Gene oder zu einer Editierung bestimmter DNA-Sequenzen führt (• Abb. 3). 
Tab. 1 Übersicht zu laufenden und geplanten klinischen Studien bei der Huntington-Krankheit

\begin{tabular}{|c|c|c|c|c|c|}
\hline Sponsor & Agens (Verabreichung) & Mechanismus & $\begin{array}{l}\text { Mutations- } \\
\text { spezifisch }\end{array}$ & $\begin{array}{l}\text { Identifier (Clinical- } \\
\text { Trials.gov) }\end{array}$ & Status der Studie \\
\hline $\begin{array}{l}\text { Hoffmann-La Roche/lonis Phar- } \\
\text { maceuticals } \\
\text { (RG6042) }\end{array}$ & ASO (intrathekal) & HTT-mRNA (total) & Nein & NCT03761849 & $\begin{array}{l}\text { Phase III } \\
\text { Aktive Rekrutierung }\end{array}$ \\
\hline $\begin{array}{l}\text { Wave Life Sciences/Takeda } \\
\text { (WVE-120101) } \\
\text { (WVE-120102) }\end{array}$ & ASO (intrathekal) & $\begin{array}{l}\text { mHTT-mRNA } \\
\text { (SNP-assoziiert) }\end{array}$ & Ja & $\begin{array}{l}\text { NCT03225833 } \\
\text { NCT03225846 }\end{array}$ & $\begin{array}{l}\text { Phase I/II } \\
\text { Aktive Rekrutierung }\end{array}$ \\
\hline $\begin{array}{l}\text { UniQure } \\
\text { (AMT-130) }\end{array}$ & miRNA (striatale Injektion ) & $\begin{array}{l}\text { HTT-mRNA (total) } \\
\text { rAAV5-miHTT }\end{array}$ & Nein & NCT04120493 & $\begin{array}{l}\text { Phase I/II } \\
\text { Aktive Rekrutierung }\end{array}$ \\
\hline $\begin{array}{l}\text { Voyager Therapeutics } \\
\text { Sanofi/CHDI } \\
\text { (VY-HTTO1) }\end{array}$ & $\begin{array}{l}\text { miRNA (kombinierte Infusion } \\
\text { Putamen and Thalamus) }\end{array}$ & HTT-mRNA (total) & Nein & IND & Präklinisch \\
\hline PTC Therapeutics & Kleine Moleküle (oral) & Prä-mRNA-Splicing & Nein & n.n. & Präklinisch \\
\hline Sangamo/Shire/Takeda & $\begin{array}{l}\text { AVV (ZFP) (parenchymatös } \\
\text { intrakraniell) }\end{array}$ & $\begin{array}{l}\text { mHTT-mRNA } \\
\text { (rAAV-ZFP-RD) }\end{array}$ & Ja & n.n. & Präklinisch \\
\hline Spark/Chop & miRNA (Infusion Putamen) & $H T T$-mRNA (total) & Nein & n.n. & Präklinisch \\
\hline Novartis & Kleine Moleküle (oral) & Prä-mRNA-Splicing & Nein & IND & Präklinisch \\
\hline
\end{tabular}

Eine der ersten strategischen Fragen zur Anwendung der Geneditierung bei der HK ist, ob HTT-kodierende Sequenzen ganz eliminiert oder ob alternativ die pathologisch verlängerte CAG-Basentriplettsequenz modifiziert werden soll. In präklinischen Studien mit Fibroblasten von HK-Patienten konnte gezeigt werden, dass mithilfe des CRISPR/Cas9Systems eine präzise Exzision von CAGBasentripletts aus dem HTT-Gen erreicht werden kann [35]. Mehrere präklinische Studien zeigen, dass eine intrakranielle Verabreichung der CRISPR/Cas9-Komplexe mittels viraler Vektoren zu einer robusten, entweder allelselektiven oder nichtallelselektiven HTT-Inaktivierung führt, die in transgenen Mäusen den klinischen und neuropathologischen Phänotyp verbessert [36, 37].

\section{Verhinderung der somatischen CAG-Expansion - Induktion einer CAG-Kontraktion}

Die CAG-Expansion im HTT-Gen, die der HK zugrunde liegt, ist instabil und kann durch Zunahme der CAG-Länge $\mathrm{zu}$ einem früheren Krankheitsbeginn in der nachfolgenden Generation führen („genetische Antizipation“ durch intergenerationale CAG-Instabilität) sowie zu Variationen der CAG-Länge im
Körpergewebe („somatische CAG-Instabilität“; [39]). Es wird vermutet, dass die somatische Instabilität bzw. Verlängerung der CAG-Anzahl eine wesentliche Rolle für den Erkrankungsbeginn und die Progression der HK spielt [40]. In Post-mortem-Gewebeproben von HKPatienten und in Tiermodellen fand sich vor allem in Nervenzellen eine deutliche Zunahme der CAG-TriplettWiederholungen, welche mit dem klinischen Schwergrad der Erkrankung zu korrelieren scheint [41].

Die DNA-Reparatur-Maschinerie spielt eine entscheidende Rolle bei der somatischen CAG-Instabilität [42]. Eine pharmakologische Modulation der DNA-Reparatur-Maschinerie (z. B. eine Hemmung von MSH-3) könnte es in der Zukunft erlauben, somatische CAGExpansionen im HTT-Gen zu reduzieren bzw. zu verhindern oder gar CAGKontraktionen $\mathrm{zu}$ induzieren.

\section{Diskussion}

Die vielversprechenden neuen Therapieansätze zur Behandlung der HK führen zu verschiedenen komplexen Fragen: Wer sollte wann mit welcher Therapie und welcher Applikationsstrategie behandelt werden?
Es liegt nahe, zu denken, dass die beste klinische Wirkung bei einem frühen Behandlungsbeginn $\mathrm{zu}$ erwarten ist, noch bevor es zu einem ausgeprägten Neuronenverlust gekommen ist. Es ist gegenwärtig allerdings ungeklärt, zu welchem Zeitpunkt die Behandlung eingeleitet werden muss, damit der Krankheitsverlauf in alltagsrelevanter Weise beeinflusst werden kann. Ebenso ist noch nicht bekannt, inwieweit HK-induzierte Schädigungen bei teilweiser (oder vollständiger) Repression der Nachbildung mutanter HTT-Gen-Produkte repariert werden können. Bei der HK als autosomal-dominanter Erkrankung mit vollständiger Penetranz ist eine eindeutige molekulare Diagnose bereits lange vor Manifestation klinischer Auffälligkeiten möglich. Große klinische Beobachtungsstudien wie TRACK-HD [2] und PREDICT-HD [7] legen nahe, das mindestens 10 Jahre vor der Entwicklung diagnoseweisender klinischer Zeichen reproduzierbar messbare bildgebende (magnetresonanztomographische) und unspezifische kognitive und motorische Alterationen nachweisbar sind. Allerdings setzt die Therapieentwicklung im präsymptomatischen oder prodromalen Stadium der HK voraus, dass verlässliche, interpretierbare Biomarker etabliert sind. Hier besteht noch viel Forschungsbedarf: 
Hier steht eine Anzeige.

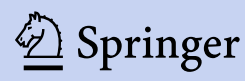


Selbst das Verständnis der Bildung, Freisetzung, Zirkulation, Verteilung und des Stoffwechsels des mHTT-Proteins im Liquor ist unvollständig.

Eine offene Frage ist, ob eine Intervention auf DNA-Ebene (z.B. mithilfe von Zinkfingerprotein-Repressor-Komplexen oder CRISPR/Cas) den Interventionen auf mRNA-Ebene überlegen ist und ob eine Degradation der Prä-mRNA einer Degradation von reifer mRNA vorzuziehen ist. Ebenso muss sich erst noch empirisch klären, ob eine allelselektive Behandlungsstrategie gegenüber einer nichtallelselektiven Behandlung klinisch relevante Vorteile bietet. Ein Pluspunkt eines nichtselektiven Ansatzes wäre, dass mit einem Therapeutikum (wie Ionis$\mathrm{HTT}_{\mathrm{RX}}$ ) die gesamte von HK betroffene Population behandelt werden könnte. SNP-basierte, allelselektive Strategien sind nur für definierte Subpopulationen denkbar. Allerdings liegen noch keine Langzeitbeobachtungen vor, die es erlauben würden, abzuschätzen, wie gut und bis zu welcher Grenze eine Reduktion des physiologischen HTT auf Dauer toleriert wird. Erst durch Langzeitbeobachtungen wird klar werden, ob bei nichtallelselektiven Ansätzen möglicherweise über eine $\mathrm{zu}$ ausgeprägte Suppression des physiologischen HTT („loss of function“) Nebenwirkungen oder Verschlechterungen des klinischen Zustandes resultieren. Entscheidend zur Vermeidung solcher unerwünschten Wirkungen dürfte die angemessene Dosierung nichtallelselektiver Therapeutika sein.

\section{》) Vektorbasierte Therapie ermöglicht einen potenziell lebenslang anhaltenden Effekt}

Eine weitere Hürde für die Behandlung mit ASOs für neurologische Erkrankungen ist die Notwendigkeit einer regelmäBig wiederholten intrathekalen (IT-)Applikation. Es ist zudem gegenwärtig ungeklärt, ob lumbale IT-Injektionen erlauben, alle relevanten Gehirnregionen mit dem Wirkstoff in therapeutisch wirksamen Konzentrationen zu erreichen. Auch für gentherapeutische Ansätze mit stereotaktischer intraparenchymatöser Ap- plikation viraler Vektoren ist gegenwärtig das effektive Transduktionsvolumen unbekannt. Ein konzeptueller Vorteil der vektorbasierten Therapie ist es, dass dank der Persistenz des episomalen Kargos ein langanhaltender, potenziell lebenslanger Effekt erhofft werden kann.

Da die Pathologie der HK nicht auf das Gehirn und im Gehirn auf die Basalganglien beschränkt ist [25] und die ubiquitäre Expression von HTT eine Multisystempathologie nahelegt, ist gegenwärtig ungeklärt, ob eine Behandlung des ZNS für eine alltagsrelevante Verlaufsbeeinflussung ausreicht und welche Vorteile eine systemische Behandlung hätte. Die Entwicklung oral verfügbarer Spleißmodulatoren wird es in naher Zukunft erlauben, diese Fragen zu beantworten.

\section{Ausblick}

Neue therapeutische Ansätze bei der HK eröffnen uns heute die Möglichkeit, den Krankheitsverlauf bereits auf den ersten pathogenetischen Schritten zu modulieren, d.h. durch Ansätze auf Ebene der DNA und der mRNA. Das ultimative Ziel dieser therapeutischen Ansätze ist es, durch eine Behandlung bereits im präklinischen Stadium der HK den klinischen Erkrankungsbeginn in ein höheres Lebensalter zu verschieben oder die Manifestation der Krankheit sogar vollständig zu verhindern. Die dynamische Entwicklung innovativer Behandlungsansätze stellt die bestehenden Versorgungszentren für Patienten, die an der HK leiden, vor neue, große Herausforderungen und erfordert die Umsetzung innovativer Kooperationsmodelle, um dem künftig deutlich zunehmenden Bedarf an geeigneten Behandlungsstrukturen gerecht werden zu können.

\section{Fazit für die Praxis}

- Bisher ist keine Therapie etabliert, die das unaufhaltbare Fortschreiten der HK modifizieren kann. Mehrere, vielversprechende therapeutische Behandlungsansätze reduzieren die Belastung des Gehirns durch mutante HTT-Gen-Produkte und wecken dadurch die Hoffnung, den Verlauf der Erkrankung verlangsamen zu können.

- Mehrere Ansätze sind entwickelt worden, die Nachbildung mutanter HTT-Gen-Produkte zu reduzieren. Die größte Erfahrung besteht derzeit mit intrathekal verabreichten ASOs: das ASO RG6042 reduzierte bei Patienten im frühen Stadium der HK dosisabhängig die Konzentration des mutanten HTT im Liquor cerebrospinalis.

- Mittels rekombinanten Adeno-assoziierte Viren als Vektoren könnte mit einer einmaligen Behandlung eine langfristige Reduktion mutanter HTT-Gen-Produkte erreicht werden. Sicherheit, Verträglichkeit und Wirksamkeit werden derzeit in klinischen Studien untersucht.

- Präklinisch werden derzeit verschiedene Ansätze zur Reduktion von mutantem HTT untersucht, darunter oral verfügbare Moleküle, die den Spleißvorgang der Prä-mRNA verändern.

\begin{tabular}{l} 
Korrespondenzadresse \\
$\begin{array}{l}\text { Prof. Dr. } \\
\text { G. B. Landwehrmeyer } \\
\text { Abteilung Neurologie, } \\
\text { Universitätsklinikum Ulm } \\
\text { Oberer Eselsberg 45/1, } \\
\text { 89081 Ulm, Deutschland } \\
\text { bernhard.landwehrmeyer@uni- } \\
\text { ulm.de }\end{array}$ \\
\hline
\end{tabular}

\section{Einhaltung ethischer Richtlinien}

Interessenkonflikt. Die Autorinnen und Autoren weisen auffolgende Beziehungen hin: A. Mühlbäck: Ihre Institution, die Abteilung Neurologie der Universität Ulm, hat im Kontext klinischer Medikamentenprüfungen Zuwendungen von folgenden Unternehmen erhalten: CHDI Foundation, Hoffmann-La Roche, Isis (IONIS) und Teva. Vortragshonorar von Desitin. Nichtfinanzielle Unterstützung (Erstattung von Reise- und Unterbringungskosten) von Actelion, EHDN sowie ein Honorar für wissenschaftliche Beratung von TEVA. K.S. Lindenberg: Ihre Institution, die Abteilung Neurologie der Universität UIm, hat im Kontext klinischer Medikamentenprüfungen Zuwendungen von folgenden Unternehmen erhalten: CHDI Foundtation, Hoffmann-La Roche, Isis (IONIS) und Teva. C. Saft: institutionelle Kompensation zur Durchführung von Studien, Erstattung von Reise- und Unterbringungskosten von IONIS Pharmaceuticals und Roche AG. Honorare für Vorträge von Teva Pharma GmbH, nichtfinanzielle 
Unterstützung (Erstattung von Reise- und Unterbringungskosten) und institutionelle Kompensation im Kontext der ENROLL-HD Studie (CHDI), PRIDE-HD (TEVA), LEGATO (TEVA), und Amaryllis (Pfizer), sowie Gelder zur Durchführung von Forschung von Biogen (alle außerhalb des vorliegenden Manuskripts). Angestellter bei der Neurologischen Klinik des Katholischen Klinikums Bochum. J. Priller: Studienteilnahme bei EHDN, CHDI, lonis/Roche, Reisekostenübernahme bei Investigator meetings, Vortragshonorar von Desitin. Keinen direkten Interessenskonflikt wegen Mitgliedschaft bei der DGPPN, DGGPP, DGBP, DZNE, IGSLI. Patent bei Epomedics, eine wissenschaftliche Kooperation bei Neurimmune und eine Beratertätigkeit bei Axon. Angestellt bei der Charité Berlin in der Klinikfür Psychiatrie und Psychotherapie. G.B. Landwehrmeyer: Drittmittel von folgenden Geldgebern/Einrichtungen: CHDI, EU (FP6\&7), BMBF, JPND und DFG. Wissenschaftliche Beratung bzw. Mitgliedschaft in einem, Scientific Advisory Board' für affiris, AOP Orphan, Bayer, CHDI Foundation, Desitin, Hoffmann-LaRoche, Lundbeck, Isis (IONIS), NeuraMetrix, Novartis, PTC, Sage Therapeutics, Teva, Triplet TX, Wave. Im Kontext klinischer Medikamentenprüfungen hat seine Institution, die Abteilung Neurologie der Universität UIm, Zuwendungen von Allergan, lonis, Hoffmann-LaRoche, Pfizer, Teva. Royalties von Oxford University Press.

Für diesen Beitrag wurden von den Autoren keine Studien an Menschen oder Tieren durchgeführt. Für die aufgeführten Studien gelten die jeweils dort angegebenen ethischen Richtlinien.

Open Access Dieser Artikel wird unter der Creative Commons Namensnennung 4.0 International Lizenz veröffentlicht, welche die Nutzung, Vervielfältigung, Bearbeitung, Verbreitung und Wiedergabe in jeglichem Medium und Format erlaubt, sofern Sie den/die ursprünglichen Autor(en) und die Quelle ordnungsgemäß nennen, einen Link zur Creative Commons Lizenz beifügen und angeben, ob Änderungen vorgenommen wurden.

Die in diesem Artikel enthaltenen Bilder und sonstiges Drittmaterial unterliegen ebenfalls der genannten Creative Commons Lizenz, sofern sich aus der Abbildungslegende nichts anderes ergibt. Sofern das betreffende Material nicht unter der genannten Creative Commons Lizenz steht und die betreffende Handlung nicht nach gesetzlichen Vorschriften erlaubt ist, ist für die oben aufgeführten Weiterverwendungen des Materials die Einwilligung des jeweiligen Rechteinhabers einzuholen.

Weitere Details zur Lizenz entnehmen Sie bitte der Lizenzinformation auf http://creativecommons.org/ licenses/by/4.0/deed.de.

\section{Literatur}

1. Macdonald M (1993) A novel gene containing a trinucleotide repeat that is expanded and unstable on Huntington's disease chromosomes. Cell 72(6):971-983

2. Tabrizi SJ et al (2009) Biological and clinical manifestations of Huntington's disease in the longitudinal TRACK-HD study:cross-sectional analysis of baseline data. Lancet Neurol 8(9):791-801

3. Rawlins MD et al (2016) The prevalence of Huntington's disease. Neuroepidemiology 46(2):144-153

4. Squitieri Fet al (2016) Epidemiology of Huntington disease: first post-HTT gene analysis of prevalence in Italy. Clin Genet 89(3):367-370
5. Ross CA, Tabrizi SJ (2011) Huntington's disease: from molecular pathogenesis to clinical treatment. Lancet Neurol 10(1):83-98

6. Marder K et al (2000) Rate of functional decline in Huntington's disease. Neurology 54(2):452-452

7. Paulsen JS et al (2014) Clinical and biomarker changes in premanifest Huntington disease show trial feasibility: a decade of the PREDICT-HD study. Front Aging Neurosci 6:78

8. Tabrizi SJ et al (2019) Targeting Huntingtin expression in patients with Huntington's disease. NEngl J Med 380(24):2307-2316

9. Arrasate M, Finkbeiner S (2012) Protein aggregates in Huntington's disease. Exp Neurol 238(1):1-11

10. DiFiglia M et al (1997) Aggregation of Huntingtin in neuronal Intranuclear inclusions and dystrophic neurites in brain. Science 277(5334):1990-1993

11. Neueder A et al (2017) The pathogenic exon 1 HTT protein is produced by incomplete splicing in Huntington's disease patients. Sci Rep 7(1). https:// doi.org/10.1038/s41598-017-01510-z

12. Zuccato C, Valenza M, Cattaneo E (2010) Molecular mechanisms and potential therapeutical targets in Huntington's disease. Physiol Rev 90(3):905-981

13. Yamamoto A, Lucas JJ, Hen R (2000) Reversal of neuropathology and motor dysfunction in a conditional model of Huntington's disease. Cell 101(1):57-66

14. Wang $\mathrm{N}$ et al (2014) Neuronal targets for reducing mutant huntingtin expression to ameliorate disease in a mouse model of Huntington's disease. Nat Med 20(5):536-541

15. Caron NS, Dorsey ER, Hayden MR (2018) Therapeutic approaches to Huntington disease: from the bench to the clinic. Nat Rev Drug Discov 17(10):729-750

16. Khvorova A, Watts JK(2017)The chemical evolution of oligonucleotide therapies of clinical utility. Nat Biotechnol 35(3):238-248

17. Pulst SM (2019) Antisense therapies for neurological diseases. Nervenarzt 90(8):781-786

18. Silva AC et al (2019) Antisense oligonucleotide therapeutics in neurodegenerative diseases: the case of polyglutamine disorders. Brain 143(2):407-429. https://doi.org/10.1093/brain/ awz328

19. Pfister EL et al (2009) Five siRNas targeting three SNPs may provide therapy for threequarters of Huntington's disease patients. Curr Biol 19(9):774-778

20. Kay C et al (2015) Huntingtin haplotypes provide prioritized target panels for allele-specific silencing in Huntington disease patients of European ancestry. Mol Ther 23(11):1759-1771

21. Bennett CF, Swayze EE (2010) RNA targeting therapeutics: molecular mechanisms of antisense oligonucleotides as a therapeutic platform. Annu Rev Pharmacol Toxicol 50:259-293

22. Geary RS et al (2015) Pharmacokinetics, biodistribution and cell uptake of antisense oligonucleotides. Adv Drug Deliv Rev 87:46-51

23. Alterman JF et al (2019) A divalent siRNA chemical scaffold for potent and sustained modulation of gene expression throughout the central nervous system. Nat Biotechnol 37(8):884-894

24. Kurosaki T,PoppMW, MaquatLE (2019) Qualityand quantity control of gene expression by nonsensemediated mRNA decay. Nat Rev Mol Cell Biol 20(7):406-420

25. Carroll JB et al (2015) Treating the whole body in Huntington's disease. Lancet Neuro 14(11):1135-1142

26. Peltz SW et al (2009) Targeting post-transcriptional control for drug discovery. RNA Biol 6(3):329-334
27. Stanek LM et al (2014) Silencing mutant Huntingtin by adeno-associated virus-mediated RNA interference ameliorates disease manifestations in the YAC 128 mouse model of Huntington's disease. Hum Gene Ther 25(5):461-474

28. Cambon K et al (2017) Preclinical evaluation of a lentiviral vector for Huntingtin silencing. Mol Ther 5:259-276

29. Podsakoff G, Wong KK Jr., Chatterjee S (1994) Efficient gene transfer into nondividing cells by adeno-associated virus-based vectors. J Virol 68(9):5656-5666

30. Evers MM et al (2018) AAV5-miHTT gene therapy demonstrates broad distribution and strong human mutant Huntingtin lowering in a Huntington's disease minipig model. Mol Ther 26(9):2163-2177

31. Pfister EL et al (2018) Artificial miRNas reduce human mutant Huntingtin throughout the striatum in a transgenic sheep model of Huntington's disease. Hum Gene Ther 29(6):663-673

32. Franich NR et al (2008) AAV vector-mediated RNAi of mutant Huntingtin expression is neuroprotective in a novel genetic rat model of Huntington's disease. Mol Ther 16(5):947-956. https://doi.org/ $10.1038 / \mathrm{mt} .2008 .50$

33. Jinek M et al (2012) A programmable dual-RNAguided DNA endonuclease in adaptive bacterial immunity. Science 337(6096):816-821

34. Cong L et al (2013) Multiplex genome engineering using CRISPR/Cas systems. Science 339(6121):819-823

35. Dabrowska M et al (2018) Precise Excision of the CAG Tract from the Huntingtin Gene by Cas9 Nickases. Front Neurosci 12. https://doi.org/10. 3389/fnins.2018.00075

36. Monteys AMetal (2017) CRISPR/Cas9 editing of the mutant Huntingtin allele in vitro and in vivo. Mol Ther 25(1):12-23

37. Yang $S$ et al (2017) CRISPR/Cas9-mediated gene editing ameliorates neurotoxicity in mouse model of Huntington's disease. J Clin Invest 127(7):2719-2724

38. Redman M et al (2016) What is CRISPR/Cas9? Arch Dis Child 101(4):213-215

39. Kennedy $L$ et al (2003) Dramatic tissue-specific mutation length increases are an early molecular event in Huntington disease pathogenesis. Hum Mol Genet 12(24):3359-3367

40. Lopez Castel A, Cleary JD, Pearson CE (2010) Repeat instability as the basis for human diseases and as a potential target for therapy. Nat Rev Mol Cell Biol 11(3):165-170

41. Shelbourne PF et al (2007) Triplet repeat mutation length gains correlate with cell-type specific vulnerability in Huntington disease brain. Hum Mol Genet 16(10):1133-1142

42. GEM-HD Consortium (2019) CAG Repeat Not Polyglutamine Length Determines Timing of Huntington's Disease Onset. Cell 178(4):887-900.e1 